\title{
Management of multiple rib fractures-results from a Major Trauma Centre with the review of the existing literature
}

\author{
Chowdhury D. ${ }^{1 *}$, Okoh P. ${ }^{2}$, Dambappa $\mathrm{H}^{3}$ \\ DOI: https://doi.org/10.17511/ijmrr.2020.i06.01
}

1* D. Chowdhury, Specialty Registrar, Emergency Medicine, Royal Preston Hospital, England, United Kingdom.

2 P. Okoh, Specialty Registrars, Emergency Medicine, Royal Preston Hospital, England, United Kingdom.

3 H. Dambappa, Specialty Registrars, Emergency Medicine, Royal Preston Hospital, England, United Kingdom.

Introduction: Rib fractures are amongst the most common fractures following major trauma presenting to the Emergency Department. The impact of rib fractures is even more significant in the patient with underlying chronic respiratory conditions. Aim: To assess our current management of rib fractures at our trauma center. Method: The current study collected our data from the TARN Registry primarily focussing on patients with multiple rib fractures. The main components were the analgesic requirement of our patients. The number of rib fracture stabilization procedures and the average number of ribs fixed were also studied. Results: The data were collected retrospectively over 12 months. There were 313 patients identified as having chest wall injuries. From the data, $41.9 \%$ (131) of patients were over the age of 65 years. A significant proportion of our patients $34.5 \%$ (108) were noted to have multiple rib fractures ( $>3$ Ribs). It was noted that $3 \%$ (9) of the 313 patients required operations. The average of the patients included in the study was noted to be 61 years with an age range of 17-92 years. Conclusion: The mainstay management of rib fracture is a provision of adequate analgesia and the prevention of respiratory complications that can all stem from poor ventilatory function amongst other patient factors and injury patterns. Through the decades, surgical stabilization has gained pace and has found its niche in the management of rib fractures.

Keywords: Rib Fracture, Trauma, Pain Management

\section{Corresponding Author}

D. Chowdhury, Specialty Registrar, Emergency Medicine, Royal Preston Hospital, England, United Kingdom.

Email: dc7740.2007@my.bristol.ac.uk

\section{How to Cite this Article}

Chowdhury D, Okoh P, Dambappa H. Management of multiple rib fractures-results from a Major Trauma Centre with the review of the existing literature. Int J Med Res Rev. 2020;8(6):379-385.

Available From

https://ijmrr.medresearch.in/index.php/ijmrr/article/ view/1227
To Browse

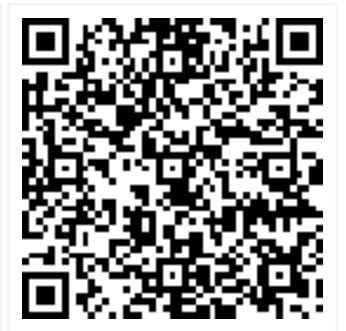

Manuscript Received 2020-11-18

Conflict of Interest No 2020-11-28

Funding Nil
Review Round 2 2020-12-04

Ethical Approval Yes
Review Round 3

Plagiarism X-checker $7 \%$
Accepted 2020-12-08

Note

(C) 2020 by D. Chowdhury, P. Okoh, H. Dambappa and Published by Siddharth Health Research and Social Welfare Society. This is an Open Access article licensed under a Creative Commons Attribution 4.0 International License https://creativecommons.org/licenses/by/4.0/ unported [CC BY 4.0]. 


\section{Introduction}

The main respiratory complications that arise from fractured ribs are multifactorial. It is well known that inadequate pain management in these patients leads to complications arising from poor ventilatory function. With an already compromised underlying pulmonary system, this compounds the effect on an already failing system leading to significant pulmonary complications. Rib fractures in the form of flail segments pose a particular problem in this regard.

When one considers an elderly patient with limited Functional Residual Capacity with an underlying diagnosis of chronic obstructive airway disease, the addition of the presence of a flail segment will invariably lead to respiratory embarrassment either acutely and /or lead to pulmonary complications in the form of basal atelectasis and pneumonia.

The main determinants of the rib score generally underpin the number of ribs that are fractured as well as the age of the patient directly correlate with morbidity and mortality. The traditional rib score is the product of the number of breaks and the sides with the addition of the age factor. Our rib score is more detailed and encompasses several other variables that have been thought to indicate a more accurate picture of mortality and morbidity.

It is recognized that if there are four or more fractures that are associated with a higher mortality rate and if there are 7 or more fractures then the mortality rate can be as high as $29 \%$. In the presence of the flail segment, the mortality rate is noted to be higher at $33 \%$ perhaps due to further respiratory embarrassment stemming from the paradoxical movement of the ribs on respiration $[1,2]$.

\section{Methods and Material}

The data collected from the TARN (Trauma Audit Research Network) registry with individual case reviews over a consecutive period of 12 months, studying patients with rib fractures.

This was done in our Major Trauma Centre in the North West of England.

Inclusion criteria for the study were all patients with rib fractures irrespective of whether it was the primary or secondary diagnosis. The individual patients were tabulated based on their ISS (Injury Severity Score).
Exclusion criteria were any patients who died followed transfer to the major trauma ward. The individual patients were studied and subgroup analysis was done on rib fixation. The average number of ribs fixed were also studied with the length of stay in the hospital included in the analysis.

The patients were followed up until the time of discharge to assess for any complications. With regards to the subgroup analysis of the complications, the research group is currently evaluating this and our findings will be published in a follow-up article. Also, the analgesic requirement of the individual patients was studied and tabulated according to their needs.

The current study noted that there was an overlap between patients receiving an epidural and the group receiving opioids. Further subgroup analysis would be a feature in future articles comparing the effect on the individual analgesic modality. There were no ethical concerns raised during the process of data collection as all the data was anonymized.

\section{Results}

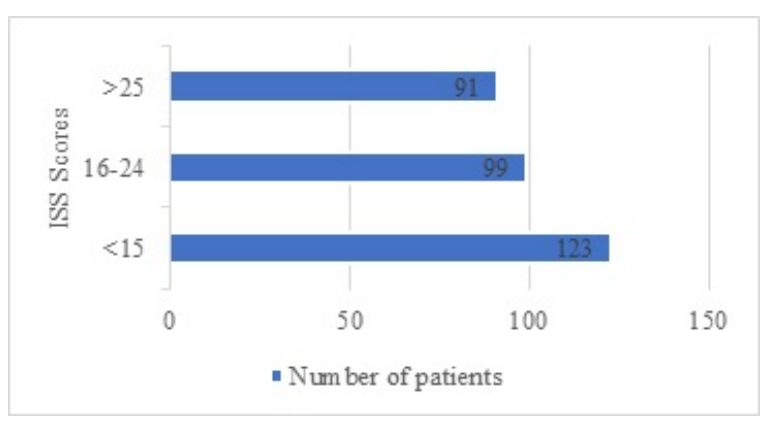

Fig-1: Number of patients included in the study and their ISS.

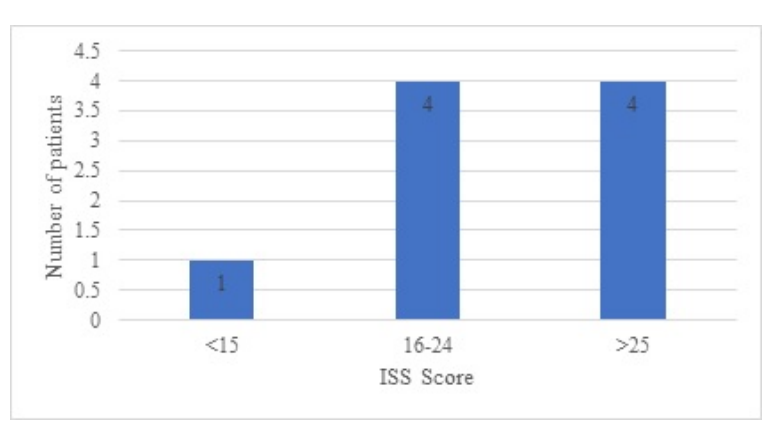

Fig-2: The number of patients requiring chest wall fixations and their ISS

Of the 9 patients that required chest fixations, the ISS score is noted to be within the high severity between 16-25. 
This indicates that the most severely injured patients required operative management of their chest wall injuries.

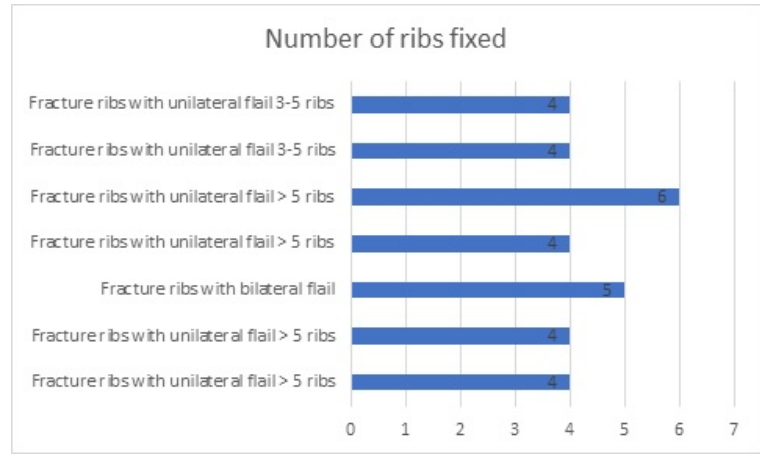

Fig-3: The number of ribs fixed and their indication.

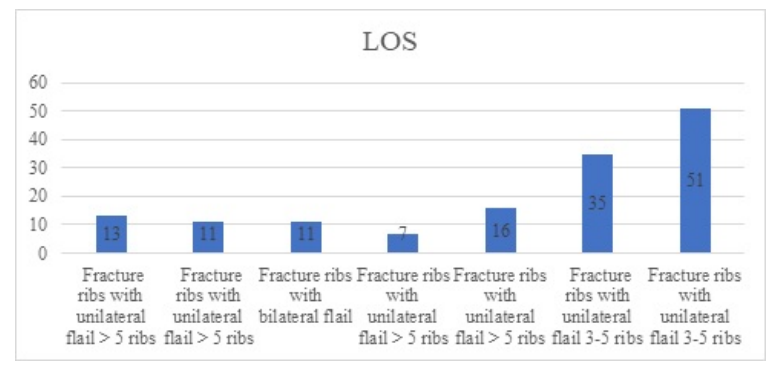

Fig-4: Indications and Length of stay in days (LOS)

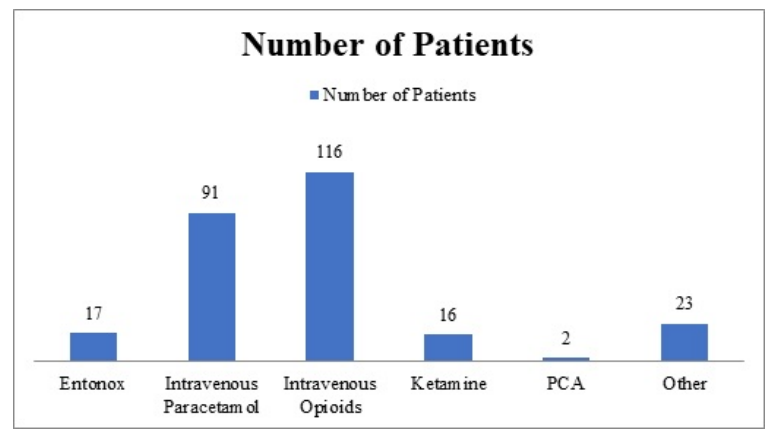

Fig-5: The different analgesic modalities

Both the groups of Intravenous opioids and PCA highlight the same group of patients. The data were collected retrospectively for over 12 months. There were 313 patients identified as having chest wall injuries. From the data, $41.9 \%$ (131) of patients were over the age of 65 years. A significant proportion of our patients $34.5 \%$ (108) were noted to have multiple rib fractures ( $>3$ Ribs). It was noted that $3 \%$ (9) of the 313 patients required operations. The average of the patients included in the study was noted to be 61 years with an age range of 17-92 years. From the data analysis, it is clear that the cases that underwent operative fixation had on average 4-5 ribs. This data is compatible with the pre-existing studies that demonstrate a similar clinical picture.

\section{Discussion}

\section{Multiple Rib Fracture Pain Management Algorithm}
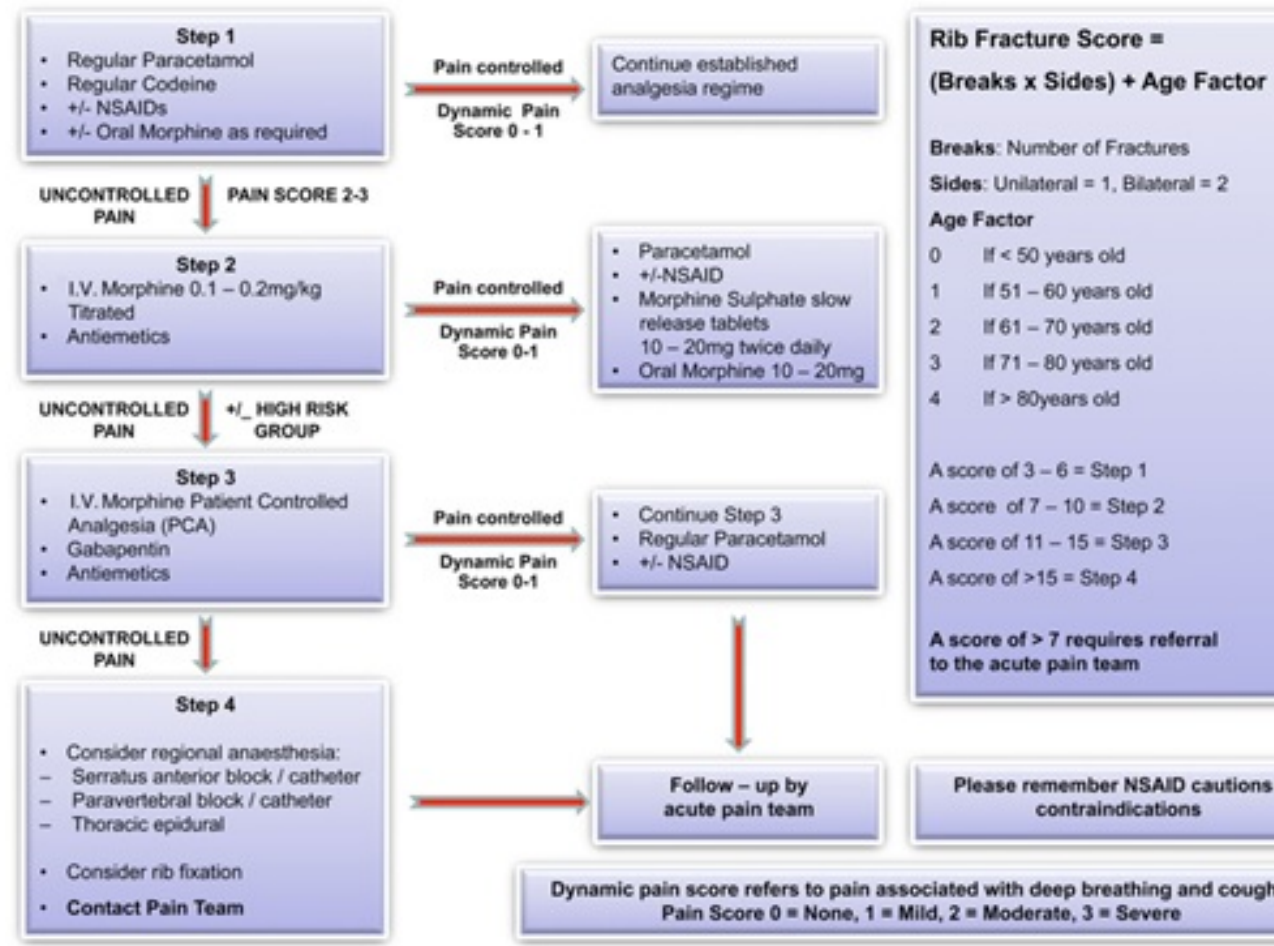
contraindications 
Fig-6: The traditional multiple rib fracture pain management algorithm

Traditional Rib Score [3]

Rib fracture Score $=($ Breaks $\times$ Sides $)+$ Age Factor

Rib Score used in our Trust [4]

Age +1 for every 10 years from aged 10

Ribs +3 for each fracture

Chronic lung disease +5

Existing anti-platelet or anti-coagulant use +4

$\mathrm{SaO2}+2$ for each $5 \%$ decrease in saturation below $95 \%$

Outcome of scoring

Score $0-10=$ CONSERVATIVE

Simple oral analgesia

? safe to discharge home. Review in the knowledge of age and comorbidities.

If pain persists or increases add in a PCA

Score 11-20 = PROGRESSIVE

PCA +/- adjuncts

If unable to take deep breaths or cough consider an epidural or paravertebral catheter

Score 21-30 = AGGRESSIVE

Epidural or paravertebral catheter

Also, consider intrapleural and intercostal blocks

If CPAP indicated for respiratory support level two bed required

Score $>31=$ EMERGENT

Epidural or paravertebral catheter

Do not site epidural in a ventilated patient until they are suitable for extubation

As it can be seen that the rib score that the current study use in our Trust is more detailed and incorporates several important patient characteristics that have a direct correlation to patient outcomes. As explained earlier that if the patient has underlying chronic lung disease, any traumatic injuries to the chest would lead to impaired ventilator function leading to complications.
The importance of anti-platelet or anticoagulant use would signify if there were concerns about underlying haemothorax/ bleeding from the fractured ribs. Reduced oxygen saturations acutely would directly correlate with inadequate oxygenation and would indirectly indicate that ventilation to the poorly aerated parts of the lung would be compromised.

Having a more detailed scoring system and associated management plan could potentially support the less experienced clinician. The true impact of the introduction of the revised rib fracture scoring system remains to be seen. The implementation of this tool in the Emergency Department will hopefully optimize the pain management of the patients earlier on as well as identify the 'at risk' patients.

The optimization of analgesia in rib fracture patients is challenging due to varied responses to analgesia bearing in mind the patients underlying comorbidities. The associated respiratory depression with the use of intravenous can be significant and needs to be taken into consideration when administering the same in a patient with impaired ventilatory function. In the review article by May [5] et al., the various regional block techniques have been described and these have proved to be useful in clinical practice. It is perhaps the early recognition that pain has a significant physiological effect on the patient's wellbeing and eventually outcomes.

\section{Optimal management of rib fractures}

The surgical management of rib fractures has been debatable and there is often a shared responsibility between orthopedic, traumatologist, and cardiothoracic surgeons. In the United Kingdom, orthopedic surgeons undertake rib stabilization. There have been studies [6] that have suggested that a combination of multiple rib fractures and underlying pulmonary contusions would be a contraindication for operative intervention. There also suggestions that patients with severe pulmonary contusions would not be suitable candidates for surgical fixation. However, currently, no imaging modality can accurately grade the degree of pulmonary contusion.

A comprehensive meta-analysis done by Tohidi et al [7] noted that there was a significant reduction in the risk of death in the surgical fixation group with also reduced need for tracheostomy and chest wall deformity. 
The main confounding factor that the authors identified in the studies available were the varying indications for the procedure.

Indications for operative management [8]

Recommended: •

- $\geq 5$ rib flail chest requiring mechanical ventilation;

- Symptomatic non-union;

- Severe displacement was found during a thoracotomy for another reason.

Consider:

- $\geq 3$ rib flail not requiring mechanical ventilation;

- $\geq 3$ ribs with severely displaced fractures (bicortical displacement);

- $\geq 3$ ribs with mild to moderate displacement and $50 \%$ reduction of expected forced vital capacity percent despite optimal pain management.

Absolute contraindications: Contaminated field.

Relative contraindications:

- Severe lung contusion requiring prolonged mechanical ventilation;

- High cervical spine injury requiring mechanical ventilation.

Pain scores in isolation are not currently being considered [9] as an indication for operative intervention due to the subjectivity and the patientto-patient variability.

One of the main domains of trauma care that is poorly studied is the follow-up period. Once the patient has had their immediate management and subsequently admitted/transferred for rehabilitation there is usually a loss to follow-up. Not including these results in an incomplete data set and the true impact of any intervention. The length of time and the frequency of follow-up is a matter of debate with some authors purporting a longer period of follow-up with a reduced frequency to gain a true understanding of the intervention.

Historically, there had been an increased uptake of patients undergoing fixation of rib fractures in patients with flail segments in the 1950s in patients with respiratory failure. However, with the increase in uptake of positive pressure ventilation the need for open reduction had reduced. It is only recently that there has been renewed interest in open reduction and internal fixation in patients with multiple rib fractures.
Trials performed recently had shown significant improvement in various parameters [10]. This ranges from the incidence of respiratory infections to length of stay in ICU. One of the main differences was the improvement in forced vital capacity in patients undergoing surgical intervention compared to those that do not. The number of ribs fixed ranged commonly between 4-6 ribs in the present study this was related to the anatomical position of the fracture and accessibility to the fractures as not all ribs fractured need to be fixed [11].

The site must be accessed whereby any residual haemothorax can be drained and any empyema drained appropriately. It may be postulated that as the population increases in countries like the United Kingdom there will be an increase in uptake in rib fracture stabilization procedures. The benefit of the operative procedure in patients with less than 5 rib fractures remains to be seen. One of the main limitations that could be addressed in future studies would be to study the trends in operative management through the years.

Performing large-scale randomized controlled trials comparing patients undergoing operative vs nonoperative management is missing from the literature. The element of survivorship bias could potentially skew results as patients would have to live long enough to undergo operative intervention. The true impact of this remains to be seen as the current study sees patients with chronic respiratory conditions living longer due to improved medical management.

\section{Highlights and take-home messages}

- Optimizing analgesia remains the cornerstone in the management of rib fractures in both isolated as well as in the setting of major trauma.

- Optimizing pain management leads to reduced respiratory complications.

- There is still limited uptake for surgical stabilization of rib fractures with scope for wider involvement based on a wider criterion. This could include performing rib fixation in patients with mild pulmonary contusions. The authors do acknowledge that this radiological finding is inherently difficult to quantify.

- Through this article, the current study has introduced the concept of a new rib-fracturebased score which is more comprehensive and takes into consideration of patient's underlying co-morbidities. 


\section{Conclusion}

The mainstay management of rib fracture is the provision of adequate analgesia and the prevention of respiratory complications that can all stem from poor ventilatory function amongst other patient factors and injury patterns. Through the decades, surgical stabilization has gained pace and has found its niche in the management of rib fractures.

\section{What does the study add to the existing knowledge?}

The reader would agree that this indeed a large study that raises the scope for a detailed in-depth analysis of various factors. The current study has identified that the individual mechanism of injuries that lead to rib fractures can be studied and appropriate primary prevention management strategies implemented. Through this article, we hope the reader would appreciate the scope for a further study comparing the traditional rib score with the new proposed rib fracture score. The current study has appreciated the fact that the traditional rib fracture score does not incorporate the significant factors that significantly contribute to the outcome of the overall rib fracture management.

Through this article, we sincerely hope that the orthopedic surgeons/cardiothoracic surgeons would consider operative interventions in patients that have significant rib fractures but do not necessarily meet the currently proposed criteria. With time we hope that the surgeons would have a lower threshold for operative intervention to maximize patient outcomes. Larger multicentric randomized controlled trials are needed to demonstrate the difference in outcomes in these two groups for comparative analysis.

\section{Author's contribution}

Dr. D. Chowdhury was the lead author of this project and was involved in the final writing of this article. Dr. P. Okoh and Dr. H. Dambappa was involved in the collection, analysis of the data as well as in writing this article

\section{Acknowledgment}

The authors would like this opportunity to thank the patients and the staff involved in the care of the severely injured patients included in the present study

\section{Reference}

01. Battle C, Lovett S, Hutchings H, Evans PA. Predicting outcomes after blunt chest wall trauma- development and external validation of a new prognostic model. Crit Care. 2014;18(3)R98. doi: $10.1186 /$ cc13873 [Crossref]

02. Holcomb JB, McMullin NR, Kozar RA, Lygas MH, Moore FA. Morbidity from rib fractures increases after age 45. J Am Coll Surg. 2003;196(4)54955.

doi: $10.1016 / \mathrm{S} 1072-7515(02) 01894-X \quad$ [Crossref]

03. Nirula R, Diaz JJ Jr, Trunkey DD, Mayberry JC. Rib fracture repair- indications, technical issues, and future directions. World J Surg. 2009;33(1)14-22.

doi: $\quad 10.1007 / \mathrm{s} 00268-008-9770-y \quad$ [Crossref]

04. Nixon G, Baird E, et al. Acute Pain Management for adult patients with fractured ribs. Rib. Ref 9/2017.

[Crossref]

05. May L, Hillermann C, Patil S. Rib fracture management. Bja Education. 2016;16(1)26-32. doi: 10.1093/bjaceaccp/mkv011 [Crossref]

06. He Z, Zhang D, Xiao H, Zhu Q, Xuan Y, Su K, et al. The ideal methods for the management of rib fractures. J Thorac Dis. 2019;11(8)S1078-89. doi:10.21037/jtd.2019.04.109 [Crossref]

07. Tohidi M, O'Sullivan D, Groome P, Yach JD. Surgical fixation of multiple rib fractures and the flail chest- A Meta-analysis and review of evidence. Orthopaedic Proceedings. 2020;102$\mathrm{B}(7) 47$.

[Crossref]

08. de Moya M, Nirula R, Biffl W. Rib fixation- Who, What, When. Trauma Surg Acute Care Open. 2017;2(1)e000059.

doi: $10.1136 /$ tsaco-2016-000059 [Crossref]

09. de Moya M, Bramos T, Agarwal S, Fikry K, Janjua $S$. Pain as an indication for rib fixation- a bi-institutional pilot study. J Trauma. 2011;71(6)1750-1754.

doi: $\quad 10.1097 /$ TA.0b013e31823c85e9 [Crossref] 
10. Tanaka H, Yukioka T, Yamaguti Y. Surgical stabilization of internal pneumatic stabilization?A prospective randomized study of management of severe flail chest patients. J Trauma. 2002;52(4)727-732.

discussion 732. doi: 10.1097/00005373-20020400000020 [Crossref]
11. Nickerson TP, Thiels CA, Kim BD, Zielinski MD, Jenkins $\mathrm{DH}$, Schiller $\mathrm{HJ}$. Outcomes of complete versus partial surgical stabilization of flail chest. World J Surg. 2016;40(1)236-241 doi: 10. 1007/s00268-015-3169-3 [Crossref] 